\title{
Noncontact Capacitive Torque Sensor For Use on a Rotating Axle
}

\author{
REINOUD F. WOLFFENBUTTEL, MEMBER, IEEE, AND JENS A. FOERSTER
}

\begin{abstract}
The measurement of the torque in a mechanically loaded axle is often required for automotive power-train and engine control systems. Up to the present, torque sensing has been performed by mainly using strain gauges connected to the axle with slip rings to enable the electrical contacting. Noncontact magnetic and optical techniques have also been employed. In the proposed capacitive torque sensor, two angular displacement sensors are spaced a well-defined distance apart. The rotor of each of these capacitive displacement sensors is composed of an array of electrodes and sinewave voltages with phase angles in the sequence $0^{\circ}, 90^{\circ}, 180^{\circ}, 270^{\circ}$ applied to it. These voltages are capacitively coupled from the stator to the rotor. The stator is also equipped with a readout electrode. The phase angle of the sinewave on this readout electrode is proportional to the rotor-to-stator electrode overlapping and, thus proportional to the angular position. The phase difference between the output signals of the two angular displacement transducers is a direct measure of the twist angle and, thus of the torque in the axle. The sensor enables the noncontact torque measurement on a $10-\mathrm{mm}$ diameter steel axle in the $0-100 \mathrm{Nm}$ range.
\end{abstract}

\section{INTRODUCTION}

$\mathrm{T}$ $\mathrm{HE}$ torque/speed relation of an engine is an important parameter for fuel-consumption economy. Optimum fuel economy would be obtained by keeping the engine at the lowest possible gear during acceleration by changing the gear ratio to give increased vehicle speed instead of operating the throttle to increase the engine speed. The throttle should only be operated to increase the power at the lowest possible engine speed. Obviously, the engine speed is not a suitable parameter for adjusting the transmission in such a strategy, so the torque should be used to adjust the transmission ratio for operation within the mechanical limitations [1]. The engine crankshaft, or another axle where the torque is to be measured, is rotating, which strongly favors a noncontacting measurement of this torque. An electrical contact with the rotating axle would require the use of slip rings, which would result in a more expensive and maintenance demanding sensor system. Another boundary condition that restricts the range of the possibilities originates from structural limitations.

The required mechanical properties of the axle usually prohibit the milling of slots for placing the sensor electronics or a local stricture of the axle for realizing a larger twist angle in the sensor. The milling of slots would be

Manuscript received February 14, 1990; revised June 30, 1990.

The authors are with the Department of Electrical Engineering, Laboratory for Electronic Instrumentation, Delft University of Technology, 2628 CD Delft, The Netherlands.

IEEE Log Number 9038787. required in noncontact strain-gauge based torque measurement systems. The electric power for operation of such a sensor can be supplied to the axle by inductive means and the telemetry of sensor data is also quite feasible. However, practical constraints do usually not permit the weakening of the axle. Moreover, the relatively high engine speed would require a careful mass balancing in the axle to avoid vibrations caused by inertia. Nevertheless, such systems have been implemented in ship axles, where the mass and diameter of the drive shaft and the number of revolutions per minute permits the mounting of a strain-gauge based sensor and readout electronics on the axle. A local stricture of the axle can be used to enlarge the torque-induced twist angle. This would facilitate the measurement of the torque using two angular displacement sensors; one on either side of the stricture. As the torque-induced twist angle is inversely proportional to the fourth power of the axle diameter, a significant gain in angular displacement can be obtained, however, structural problems usually prohibit the implementation of these techniques.

The relation between the twist angle $\tau$, over an axle length $L$ in an axle of uniform diameter $D$ and a modulus of rigidity $G$, at an applied torque $T$ can be described by

$$
\tau / T=\frac{32 L}{\pi G D^{4}}[\mathrm{rad} / \mathrm{Nm}]
$$

which results for a $10-\mathrm{mm}$ thick steel axle $\left(G_{\text {steel }}=8.10^{10}\right.$ $\left.\mathrm{N} / \mathrm{m}^{2}\right)$ and a sensing distance $L=100 \mathrm{~mm}$ in: $\tau / T=$ $10^{-3} \mathrm{rad} / \mathrm{Nm}$. The torque can, therefore, be determined using two angular displacement sensors spaced a distance $L$ apart to measure this twist angle. For direct torque sensing, with an inaccuracy smaller than $1 \mathrm{Nm}$ and without stricture of the mechanical structure, displacement sensors are required with a circumferential inaccuracy smaller than $6 \mu \mathrm{m}$. The simplest differential noncontact angular displacement sensing technique is based on the mutual displacements of two flanges that are clamped on the axle spaced a certain distance apart. Optical, magnetic, and capacitive displacement sensing techniques can be applied. An optical torque sensor is shown schematically in Fig. 1. Two disks, each with a slit, are mounted on an axle a distance $L$ apart. The twist angle controls the overlapping between the slits and thus pulsewidth modulates the transmission from a LED lightsource to a photodetector. Disks are implemented for pursuing a mechanical am- 

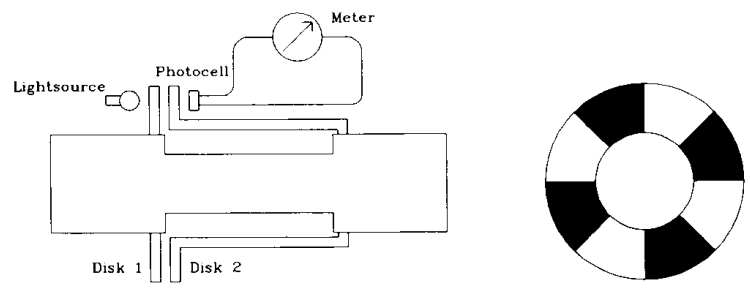

Fig. 1. Optical torque sensing using two disks with a torque-modulated slit width.

plification of the torque induced angular displacement in this sensor in order to enable torque sensing using conventional displacement sensors. The use of disks is restricted by the available space, which is limited by mechanical boundary conditions such as those imposed by bearings.

A noncontact magnetic torque sensor is possible, based on the magnetostrictive effect which is basically a straininduced magnetic field line deflection. The torque-induced compressive and tensile stresses distort the magnetic field lines as shown in Fig. 2. Applying a magnetic field in the axial direction using external coils makes it possible to measure the perpendicular field component. A second pair of coils can be used for noncontact sensing of the perpendicular field and thus enable the noncontact sensing of the torque. A disadvantage of this method is the power dissipation and the dependence of the sensitivity on the type of steel used. It is possible to get around the latter disadvantage by using the ferroelectric properties of amorphous ribbons instead of the magnetostriction of the axle itself. Groups of amorphous iron-based ribbons can be bonded to the axle with the longitudinal direction of one half of the ribbons at $45^{\circ}$ with respect to the axial direction and the other half perpendicular to that as shown in Fig. 3 [2]. When applying a torque to the axle, half of the ribbons will be subjected to tensile stress and the other half to compressive stress. The magnetostriction changes the permeability of the ribbons and causes a change in the mutual inductances between the drive coil that drives both groups of ribbons, and the two sense coils that detect the torque-induced magnetic anisotropy in the two perpendicular groups of ribbons. This method, therefore, enables a noncontact torque sensing [3], [4].

A disadvantage of the differential angular displacement method for measuring the twist angle is that the difference between the output signals of two sensors is generally used to determine the twist angle in the readout circuitry rather than the response of one differential sensor. A very interesting noncontact capacitive torque sensor that overcomes this drawback has recently been reported in literature and describes a noncontact torque sensor based on a differential capacitive displacement sensor. This sensor is shown in Fig. 4 [5] and consists of two sets of serrated teeth. One set is applied to the outside to a cylindrical tube of dielectric material. The tube is clamped around the shaft at a distance $L$ from the teeth on the axle in such
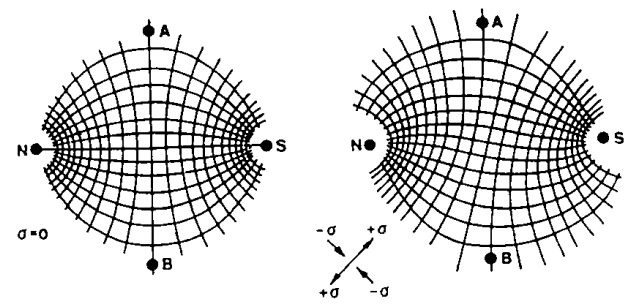

(a)

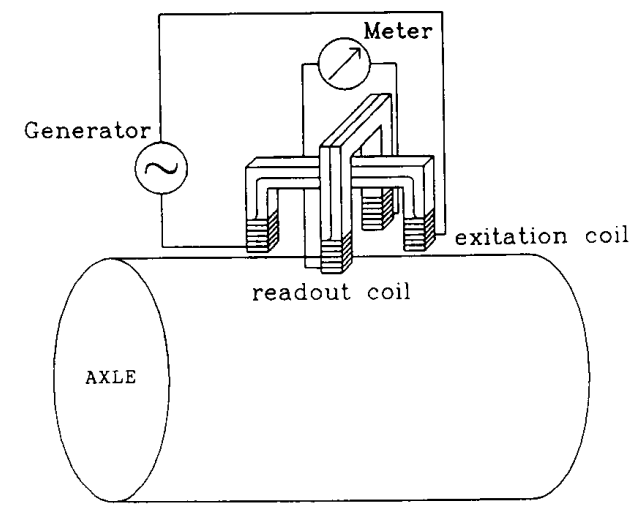

(b)

Fig. 2. Operating principle of (a) the magnetostrictive torque sensor and (b) the basic sensor structure.

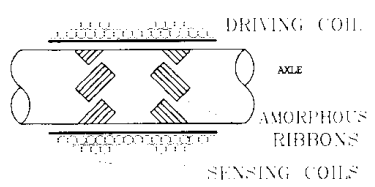

Fig. 3. Magnetic torque sensor using amorphous ribbons [2].

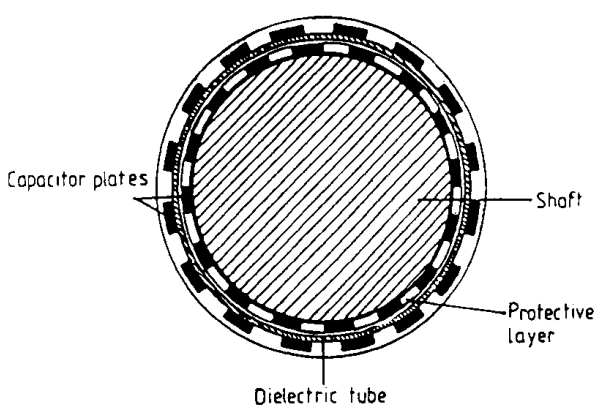

(a)

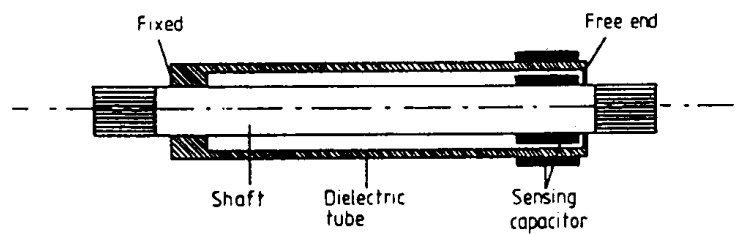

(b)

Fig. 4. Capacitive torque sensor described in [5]. (a) Cross section and (b) longitudinal section. 
a way that the teeth on the axle and those on the tube are overlapping. A twist angle results in a movement of one set of teeth with respect to the other, which enables the measurement of the torque by measuring the change in capacitance. The readout is based on the change of an $L C$ product using a grid dip principle. Although this method results in a noncontact, low-cost, and reliable sensor, it requires a rather complicated mechanical structure composed of two coaxial cylinders with a rubber bearing at the free end. Apart from this sensor, research is directed towards inductive torque sensors based on amorphous ribbons. The response of the capacitive torque sensor discussed here is solely determined by the overlap between two electrode patterns that are directly connected to their respective substrates, the axle and the casing, and is therefore based on a very simple mechanical structure which is tolerant to the spatial limitations that are imposed by e.g., bearings.

\section{The Capacitive Torque Sensor}

The capacitive torque sensor is basically a differential angular displacement sensor and is composed of two capacitive displacement sensors mounted on the axle and spaced a certain distance apart in order to enable the measurement of the twist angle. The operation of each of the capacitive displacement sensors is based on the combined capacitive coupling of four sinewaves to the readout electrode. The rotor is composed of an array of electrodes organized as a bar-space type of grating and the stator consists of a single bar-shaped readout electrode. The array of rotor electrodes are connected to sinewaves with the same amplitude, however, adjacent electrodes have a phase difference of $90^{\circ}$. In this way the repetitive phase pattern $0^{\circ}, 90^{\circ}, 180^{\circ}, 270^{\circ}$, is generated along the rotor with a periodicity over four electrodes of the array. The phase of the sinewave on the stator electrode is determined by the superimposed coupling between the individual stator electrodes and the readout bar, which depends on the mutual spacings, and is therefore a direct measure of the position of the stator with respect to the rotor [6].

The fringing fields can be disregarded in case of a rotorto-stator spacing much smaller than the width of the electrode bars and at relatively low angular velocities. For a 10 -mm diameter axle with a spacing of about $1 \mathrm{~mm}$ this implies that only one set of four driving electrodes is allowed along the circumference. An almost linear relation between the output phase and the angular position can be obtained when using a rotor pattern with a spacing equal to the bar width, $a$, and a stator electrode width equal to $((D+2 s) / D) \times(3 a)$, as shown in cross section in Fig. 5 . The electrical phase at the readout electrode changes over $360^{\circ}$ when the rotor completes one full mechanical revolution. This sensor, therefore, enables the dimensionless transduction from mechanical rotation over a certain angle into an electrical phase shift. The sensor is intended for application in the $0-100 \mathrm{Nm}$ torque range. The associated maximum angle of rotation between the two angular displacement sensors spaced a distance $L=100 \mathrm{~mm}$

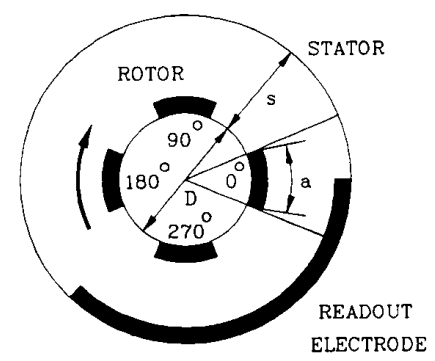

Fig. 5. Basic capacitive angular displacement sensor.

apart can easily be derived from (1) and is equal to $\tau=$ $5.7^{\circ}$. A minimum resolvable torque equal to $0.2 \mathrm{Nm}$ can be detected when using a phase meter with a $0.01^{\circ}$ resolution. This performance is more than adequate for practical torque measurement in the intended application area, however, the eventual objective of the torque sensor presented here is the integration of the sensor readout circuitry with a simple analog-to-digital (AD) converter in a single chip. This 'smart sensor' will enable on-chip phase readout with $0.1^{\circ}$ resolution without having to resort to external professional equipment, by using a phase-topulsewidth conversion to drive an integrated counter for a gated AD conversion based on counting. This miniaturization is pursued at the expense of a reduced resolution of the phase measurement and an unmodified sensor configuration would yield a torque resolution of only $2 \mathrm{Nm}$. To compensate for this detrimental effect, a differential sensor structure should be designed with an intrinsic better resolution. Such an objective can be met by changing the number of electrodes and the electrode dimensions, which results in a sensor where the effect of fringing fields can no longer be disregarded. A sensor with an improved performance is presented in the next section.

\section{IMPROVED SENSOR}

The resulting sensor structure would be composed of 16 rotor electrodes and 4 stator electrodes as shown schematically in cross section in Fig. 6. In this way a phase pattern of four times $360^{\circ}$ is generated along the circumference of the rotor. Basically, this method implies the transformation of the mechanical rotation over an angle $\tau$ into an electrical phase change equal to $4 \tau$. The four readout bars are distributed equidistant over the stator. The detected phases are, therefore, synchronous and the four readout strips can be connected in parallel in order to increase the nominal transducer capacitance and, thus, also the signal amplitude of the sinewave at the input of the readout circuitry is enhanced. This concept can be extended to $4 n$ rotor bars and $n$ stator bars. Photolithographic constraints limit the bar width, $a$, and the bar spacing to about $a_{\min }=0.4 \mathrm{~mm}$. This limits $n$ to $n_{\max }=$ $\pi(D+s) /\left(2 n \times a_{\min }\right)=(\pi \times 11) /(8 \times 0.4)=10$. This results in a torque resolution in the practical sensor exceeding $0.2 \mathrm{Nm}$, which is well within the range of the target values. Unfortunately, extreme accuracy requirements should be imposed on the tolerances and the excen- 


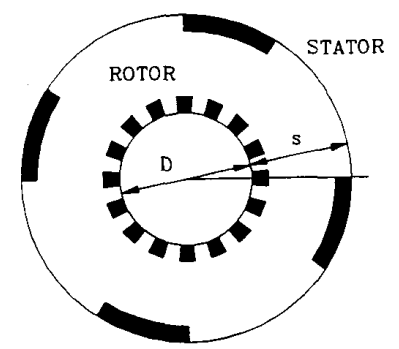

Fig. 6. Capacitive angular displacement sensor with an enhanced resolution.

tricity of the rotor-to-stator spacing, $s$, for obtaining synchronized phases at all readout bars. A cancellation of the readout signals will occur instead of reinforcement if such requirements are not met and the signal level enhancement, pursued by the synchronization, is largely undone by the tolerances in a practical structure.

For this reason a sensor is constructed with several electrical periods over the rotor circumference, however, with only one stator readout strip irrespective of the number of rotor electrodes. The bar width, $a=0.4 \mathrm{~mm}$ and the rotor-to-stator spacing $s=1 \mathrm{~mm}$. These dimensions indicate that the parallel-plate approximation is utterly inadequate for describing the sensor characteristics. The angular displacement sensor with improved resolution is basically of the incremental type. Therefore, an ambiguity can occur in the readout of extremely high torques. A small torque is indicated if the angular displacement exceeds four rotor strips. The practical torque sensor is designed to exhibit a sufficiently large safety margin.

The angular displacement sensor is composed of a coaxial stator and rotor electrode as depicted at several cross sections along the axial direction in Fig. 7. The schematic diagram of the equivalent electrical circuit is shown in Fig. 8. The values of the capacitors $C_{1}$ through $C_{4}$ depend on the angular displacement. $C_{r}$ is the return coupling capacitor between the rotor and the stator and $C_{a}$ is the capacitance between the rotor and the conductive axle. When assuming $C_{a} \gg C_{r}, C_{1}, C_{2}, C_{3}, C_{4}$, an output voltage $U_{o}$ is generated that can be described by

$$
\begin{aligned}
U_{o}= & -\frac{C_{r}}{C_{f} C_{a}}\left(\left(C_{2}-C_{4}\right) U \sin \omega t\right. \\
& \left.+\left(C_{1}-C_{3}\right) U \cos \omega t\right) .
\end{aligned}
$$

The phase of the output voltage contains the desired angular position information, hence

$$
\arg \left(U_{o}\right)=\arctan \left(\frac{C_{2}-C_{4}}{C_{1}-C_{3}}\right) .
$$

As the fringing-fields can no longer be disregarded, it is not possible to derive a simple analytical expression for the relation between $\arg \left(U_{o}\right)$ and the angle of rotation. Equation (3) has been solved numerically and the result is shown for one period of the repetitive stator pattern in Fig. 9. The torque sensor is composed of two sets of these

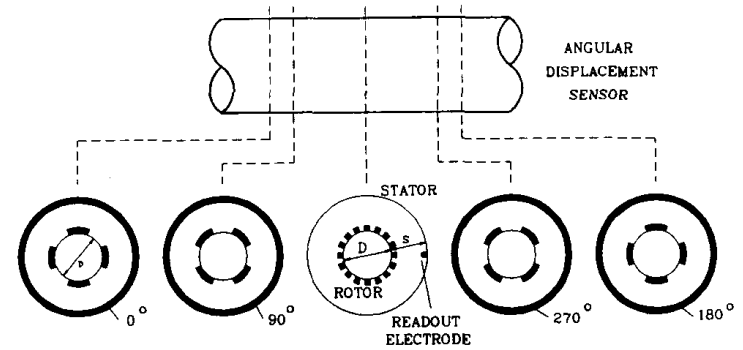

Fig. 7. Practical capacitive angular displacement sensor for different cross sections along the axial direction.

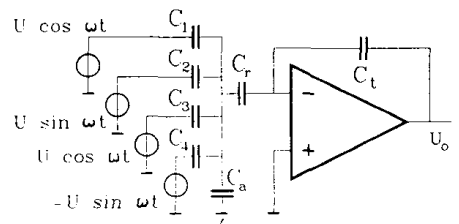

Fig. 8. Equivalent electrical circuit of the sensor capacitors with the parasitics and the readout charge amplifier.

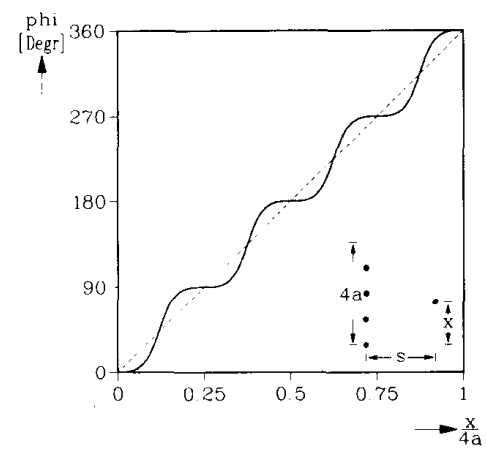

Fig. 9. Calculated response of an angular displacement sensor with a rotor-to-stator spacing, $s$, much larger than the electrode spacing, $a$. The phase angle on the readout electrode is shown versus the position $X$

angular displacement sensors spaced a well-known distance apart and $\left\{\arg \left(U_{o 1}\right)-\arg \left(U_{o 2}\right)\right\}$ is used as a measure of the torque-induced twist angle $\tau$. As arg $\left(U_{o}\right)$ is not a linear function of the angle of rotation, a modulation of $\left\{\arg \left(U_{o 1}\right)-\arg \left(U_{o 2}\right)\right\}$ occurs when measuring the torque on a rotating axle. This property prevents the direct torque measurement and the average value of $\left\{\arg \left(U_{o 1}\right)-\arg \left(U_{o 2}\right)\right\}$ is used instead. The measurement of the average of the difference in phase can easily be implemented in the readout circuits, using a phase-topulsewidth modulation and a counter for the pulsewidthto-digital conversion, by counting over the time that is needed to travel over at least one set of 4 bars. The measurement time depends on the angular velocity and gives a minimum measurement delay equal to: the time per revolution $/ n$. An improved signal-to-noise ratio can be obtained by counting over $n / m$ revolutions, however, the increased measurement time will give an extra delay 


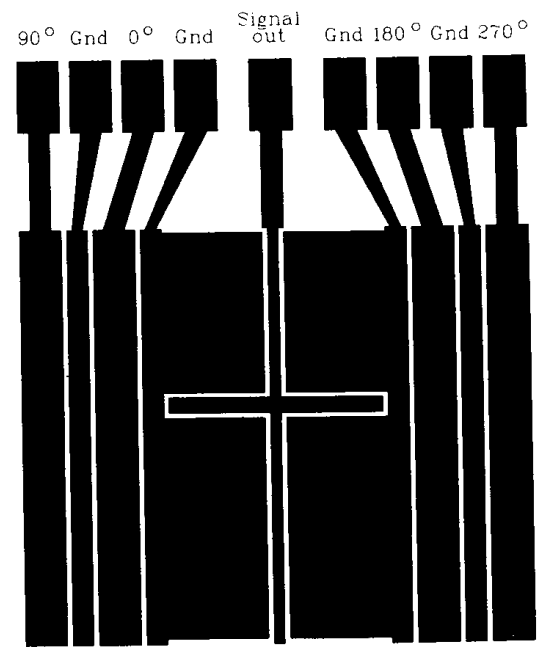

(a)

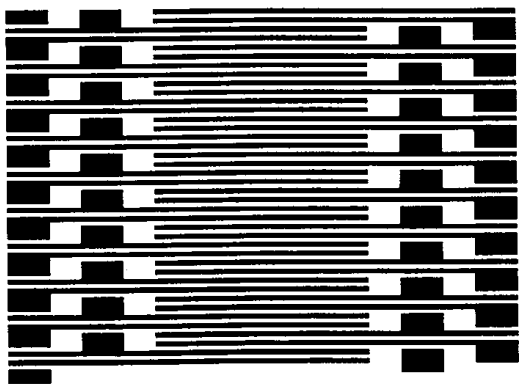

(b)

Fig. 10. (a) Stator pattern and (b) rotor pattern used for the angular displacement sensors that are implemented in the practical torque sensor

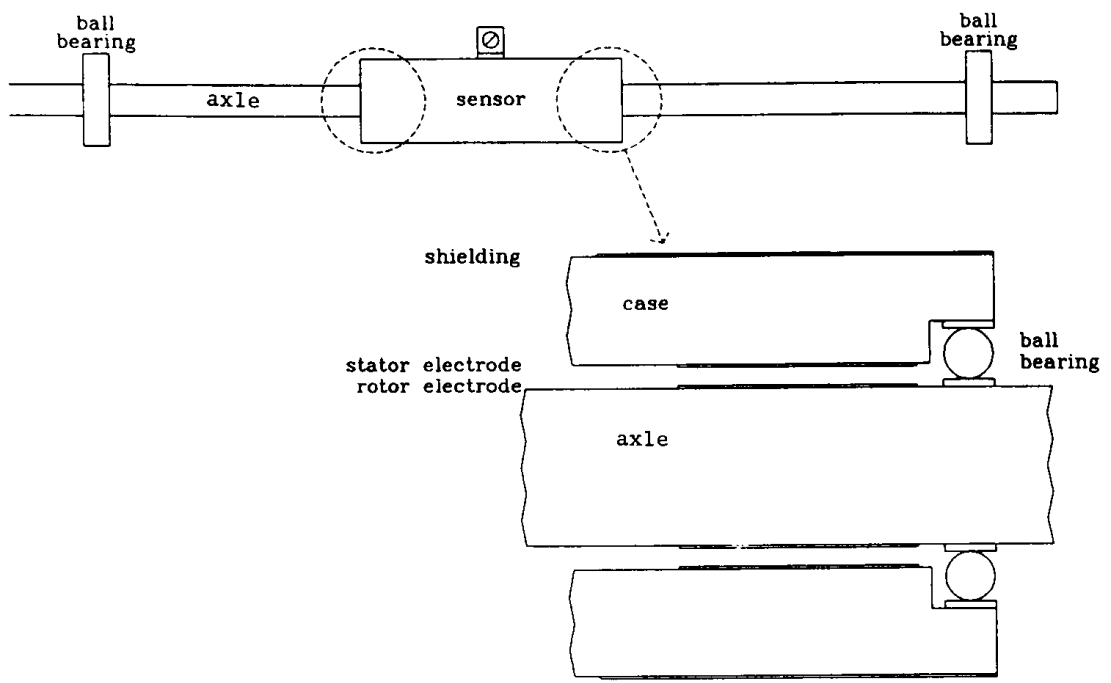

Fig. 11. Capacitive torque sensor

which might affect the stability of a closed-loop system that is based on this sensor.

The electrodes are realized on flexible printed circuit board material and are attached to their respective substrates using a special cement that is customarily used for creep-resistant bonding of strain gauges. A dielectric is required in between the rotor electrode and the axle with a thickness in the same order of magnitude as the rotorstator spacing. This measurement prevents the loss capacitance, $C_{a}$, between the interdigitized rotor electrode and the conductive axle from severely reducing the amplitude of the ac voltage before return coupling to the detector. The stator and rotor electrode patterns of the capacitive angular displacement sensor are shown in Fig. 10. The rotor pattern basically consists of a structure with large area pads for the capacitive coupling of the ac voltages to the rotor. The driving and readout electrodes are separated using a ground electrode in between to prevent direct coupling to the stator and to minimize stray-field coupling between stator and rotor.

\section{Sensor Performance}

The sensor performance for static torques has been measured using the sensor with a $10-\mathrm{mm}$ diameter steel axle that is clamped on one side, as depicted in Fig. 11. On the other side, a lever is connected to the axle. A charge amplifier has been used for the readout of the out- 


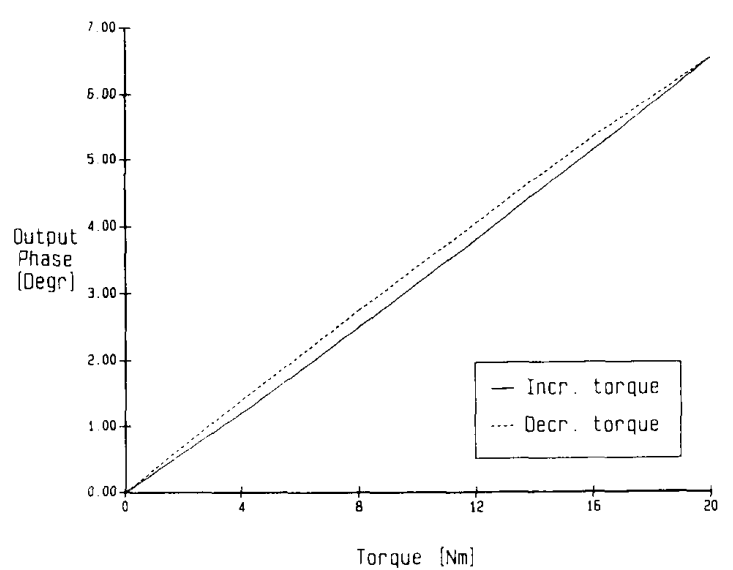

Fig. 12. Results of measurements performed on the capacitive torque sensor at increasing and decreasing torque.

put phase. A torque can be applied to the axle using standard weights connected to the end of the lever. The response of the sensor to a torque increasing from 0 to 20 $\mathrm{Nm}$ and subsequently decreasing again to $0 \mathrm{Nm}$ is shown in Fig. 12. Preliminary measurements have been performed on a rotating axis at low angular velocities. The results are in agreement with the static response curves, however, the accuracy of these measurements was not yet sufficient to give quantitative results. The measurements indicate a hysteresis. Increasing the load and subsequently reducing the load again at a certain value of the applied torque does not immediately give the initial phase angle. The error decreases after a few minutes. This behavior is characteristic for creep. The bonding technique used to cement the rotor electrode to the axle is critical for this effect and a substantial improvement can be expected when using a thick-film printing technique for the realization of the rotor electrodes. As no saturation occurs, this sensor is suitable for a wider range of torques. In the present measurement setup, it was not yet possible to apply such torques.

\section{CONCLUSions}

Noncontact capacitive torque sensing is possible based solely on the capacitive coupling between a stator and a rotor electrode pattern. A very robust and simple torque sensor can be constructed in this way. A sensitive angular displacement-to-phase angle conversion has been implemented for the readout with a sensitivity exceeding $0.3^{\circ} / \mathrm{Nm}$. Present prototypes suffer from hysteresis, which can be reduced when using a different technique for the bonding of the rotor electrode. As the phase difference between two capacitive displacement transducers is measured, the torque indication is not affected by the angular velocity of the axle. A fixed angular mismatch between the two displacement sensors gives rise to an offset, which can easily be compensated by an initial torque measurement prior to the mechanical loading of the axle. The mechanical power supplied by the axle can also be determined when using the rate of change in the phase of one of the displacement sensors as the velocity signal. Future research will be focussed on a reduction of the creep behavior and a widening of the operating range by testing different bonding techniques as well as by optimizing the electrode geometry. The research will also aim at the realization of a custom silicon chip that will contain the electronic circuitry required for the driving and the readout of the sensor.

\section{REFERENCES}

[1] M. H. Westbrook, "Sensors for automotive application," J. Phys. E: Sci. Instrum., vol. 18, pp. 751-758, 1985.

[2] I. Sasada, E. Sakai, S. Uramoto and K. Harada, "Noncontact torque sensor employing synchronized switching process," in Proc. 5th Sensor Symp., Japan, pp. 115-120, 1985.

[3] H. Hase and M. Wakamiya, "Torque sensor," in Proc. 8th Sensor Symp., Japan, pp. 279-282, 1989.

[4] Y. Nishibe, Y. Nonomura, M. Abe, K. Tsukada, M. Takeuchi, and I. Igarashi, "Real time measurement of instantaneous torque with high accuracy using magnetostrictive sensor," in Proc. 8th Sensor Symp., Japan, pp. $111-114,1989$

[5] J. D. Turner, "The development of a thick-film noncontact shaft torque sensor for automotive applications." J. Phys. E: Sci. Instrum., vol. 22, pp. 82-88, 1989.

[6] K. B. Klaassen and J. C. L. van Peppen, "Linear capacitive displacement transduction using phase readout," Sensors and Actuators, vol. 3, pp. 209-220, 1982/83. 